\title{
Robust Adaptive Watermarking Based on Image Contents Using Wavelet Technique
}

\author{
A. K. Verma \\ Electrical and Electronics Engineering Department Hindustan Institute of Technology and Management, Agra, UP, \\ India \\ Email: ajaykrverma@yahoo.com \\ C. Patvardhan \\ Electrical Engineering Department Dayalbagh Educational Institute, Agra, UP, India \\ Email: cpatvardhan@gmail.com \\ C. Vasantha Lakshmi \\ Physics and Computer Science Department Dayalbagh Educational Institute, Agra, UP, India \\ Email: cvasantha@rediffmail.com
}

\begin{abstract}
A good watermarking scheme should be able to perform equally well on all types of images irrespective of image contents because practically watermarking has to be applied to images of all types. In this paper, it is shown that in wavelet based spread spectrum technique, watermarking at level 1 decomposition is better for textured images while watermarking at level 2 decomposition is better for nontextured images to achieve maximum robustness against various types of attacks. The proposed wavelet decomposition level selection algorithm utilizes the edge histogram to classify the host image as textured or nontextured image and automatically selects the level of decomposition for robust watermarking. The use of Spread Spectrum watermarking technique and Bior6.8 wavelet, results better robustness. Performance of the proposed scheme and its relative effectiveness is demonstrated on both categories of images under different attacks.
\end{abstract}

Index Terms-Wavelets, Bior6.8, Robustness, Correlation, Edge Histogram, Level of decomposition, Image Contents.

\section{INTRODUCTION}

The rapid advancement of internet and computer technology has facilitated authorized as well as unauthorized manipulation and reproduction of digital multimedia contents. Therefore, design and development of effective digital multimedia copyright protection methods to prevent unauthorized duplication or tampering have become necessary in present time. To this end, watermarking of digital content has evolved as a possible solution. An efficient and effective watermarking technique has to satisfy at-least three major requirements i.e. Imperceptibility, Robustness and sufficient Payload amount. Simultaneously maximizing all the three together is difficult as they are noncommensurable. Therefore, finding a solution that provides satisfactory values of these parameters is a challenging task. Several watermarking algorithms are reported in the literature. Initial efforts were in the spatial domains [1, 2, 3]. Later, several improvements of frequency domain watermarking $[4,5,6]$ were reported. Among frequency domain techniques, wavelet based watermarking schemes are more attractive $[7,8,9,10,11]$ due to several advantages such as space-frequency localization and multi-resolution offered by the wavelet transform. Image contents play a very important role in deciding the performance of the watermarking process. An image may have several regions having different types of contents such as smooth (low frequencies) or textured (high frequencies). Smooth areas have low distortion resistance while textured areas can bear higher level of distortion. In the literature, several techniques are proposed which utilize image contents for effective watermarking. A DCT based watermarking method based on image contents is given in [12]. This method proposes the creation of a Just Noticeable Distortion (JND) mask which contains the JND values of each pixel. The mask is prepared on the basis of some image features such as Texture, Edges and Luminance satisfying Human Visual Systems (HVS). This method uses spread spectrum technique but results regarding quality of watermarked images and watermark extraction under various attacks are not shown. A DFT based watermarking scheme based on image contents is presented in [13]. In this scheme host image is divided into 9 sub images in $3 \times 3$. Watermark is embedded only in those sub images which are highly textured to avoid noticeable artifacts. The textured image blocks are identified by the use of Harris Corner detector. The PSNR achieved is around 40dB. The quality of recovered watermark is not shown. Only the presence and absence of watermark is highlighted. A DCT based watermarking method is given in [14]. This method prepares a mask based on image features such as 
texture, luminance, corners and edges. It embeds the watermark in those areas, which are less sensitive to human eyes. It uses decimal sequences of watermarking instead of random sequence. The PSNR achieved is in range of 35 to $38 \mathrm{~dB}$ and quality of recovered watermark is not mentioned. Another method of watermarking based on local image features is proposed in [15]. This method is based on computation of a Noise Visibility Function (NVF) that characterizes the local image properties with high texture and edge regions, where watermark can be embedded strongly without resulting visible artifacts. This method achieves PSNR in range of 25 to $34 \mathrm{~dB}$ while quality of recovered watermark is not discussed. A wavelet based watermarking method based on image contents such as texture and luminance is given in [16]. In this approach, masking is done pixel by pixel based on texture and luminance. PSNR achieved in this method is around $35 \mathrm{~dB}$ for various images. Watermark detection is shown instead of watermark recovery. A watermarking algorithm based on image contents in Ridgelet domain is proposed in [17]. In this method, image is first partitioned into small pieces. These pieces are classified as weak texture or strong texture according to the statistical properties of columns coefficients in Ridgelet Transform (RT). The middle frequencies of RT sub-bands are selected and watermarks are embedded in the higher energy directions of the pieces with strong texture which are less sensitively to human's vision. Another content based watermarking in Fourier domain is given in [18]. In this scheme, a perceptual mask is generated, which identifies both textured as well as smooth areas of the images. The embedding strength is then adjusted according to the embedding areas. The weighted PSNR is computed as quality of embedding. A hybrid image watermarking scheme based on DWT and SVD is proposed in [19]. In this approach, the edge information of an image is used to embed the watermark. In addition, the particle swarm optimization algorithm is used to search the proper value of watermark embedding strength. Experimental work shows the robustness of proposed scheme against various image processing attacks. Another watermarking algorithm robust against geometric attacks, based on image features is proposed in [20]. In this scheme, Watermark is embedded in the areas represented by the salient features of the image. It is shown that in an image, Salient features are resistive to the geometrical attacks. These salient features also provide reference points used in watermark embedding and detection.

In all these methods proposed, the emphasis is on finding a suitable mask and adjusting the embedding strength of watermark accordingly. Most of the work is reported for the non-wavelet based techniques. Even in the case of few wavelet based schemes, the proposed content based methods do not explore the role and suitability of particular wavelet function, level of decomposition etc. in watermarking. Practically, whole image may belong to either smooth or textured category. How these proposed methods would deal with such cases, is not adequately discussed.
In this paper an attempt has been made to identify the image type first on the basis of its contents and then applying suitable wavelet based watermark embedding scheme. The rest of the paper is organized as follows. Section 2 provides motivation for the work. Section 3 describes the wavelet decomposition level selection algorithm. Section 4 explains the proposed watermark embedding and watermark extraction algorithms. Section 5 shows the experimental results and some conclusions are given in section 6 followed by the references.

\section{Motivation}

It is well understood that textured images have more high frequency components at a given level of decomposition. Some higher order wavelets such as Db10, Bior6.8 etc. capture these high frequency details and produce larger wavelet coefficients as compared to smooth images. Due to large wavelet coefficients the embedding strength is not enough to maintain the impact of watermarking, which results poor watermark recovery. This is illustrated by using eight test images which are shown in figure 1. Four images of top row are smooth (non-textured) and rest four are textured. The textured images are taken from standard texture image database of Brodatz [21].

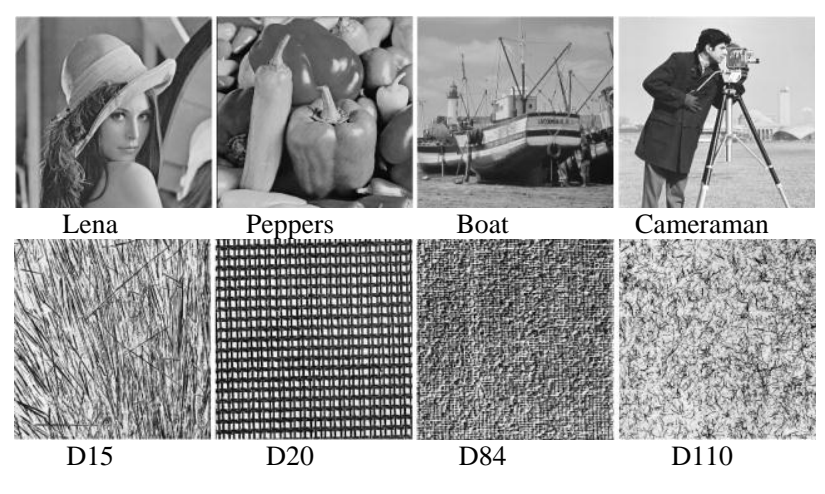

Fig 1. Textured and Non-Textured Test Images

The effect of image contents on robustness for textured and non-textured images is shown in table 1. The spread spectrum watermarkingis done on these images with embedding strength $k=2$ and Bior6.8 wavelet. The watermark is embedded in diagonal wavelet coefficients (cD). The watermark embedding and extraction algorithms used in this process are explained in detail in section 4 of this paper. The energies of diagonal wavelet coefficients (embedding plane) at level-1 (EcD1) and at level-2 (EcD2) are also shown in table 1.The energies of diagonal coefficients show the amount of texture or randomness present in the image. Although sum of energies of all the detailed coefficients can also be considered for this purpose. The values of peak signal to noise ratio (PSNR) computed between original and watermarked images are shown in table 1. To show the quality of recovered watermark, Normalized Correlation Coefficient (NC) is computed between original and recovered watermark. 
Table 1. Effect of Image contents on robustness

\begin{tabular}{lcccccr}
\hline \multirow{2}{*}{ Images } & \multicolumn{2}{c}{ Level 1 } & \multicolumn{2}{c}{ Level 2 } & \multicolumn{2}{c}{ Energy of cDs } \\
\cline { 2 - 7 } & PSNR & NC & PSNR & NC & EcD1 & EcD2 \\
\hline Lena & 30.71 & 1.00 & 37.53 & 1.00 & 6.73 & 109.33 \\
Peppers & 30.71 & 1.00 & 36.98 & 1.00 & 91.44 & 94.66 \\
Boat & 30.64 & 1.00 & 37.33 & 1.00 & 69.75 & 119.66 \\
Cameraman & 31.39 & 1.00 & 38.08 & 1.00 & 138.83 & 146.97 \\
D15 & 31.20 & 1.00 & 37.99 & 0.94 & 107.44 & 1372.80 \\
D20 & 31.37 & 1.00 & 37.98 & 0.98 & 116.14 & 660.53 \\
D84 & 31.70 & 1.00 & 38.27 & 0.96 & 116.32 & 1279.70 \\
D110 & 31.64 & 1.00 & 38.15 & 0.76 & 275.73 & 3167.70 \\
\hline
\end{tabular}

It can be clearly observed from table 1, that large wavelet coefficients (higher coefficients energies) are obtained for textured images as compared to smooth images which dilute the embedding. Therefore, poor robustness as indicated by reduced values of $\mathrm{NC}$ at level 2 , is obtained for textured images. There may be two approaches by which the robustness of watermarking can be maintained for textured images at par with smooth images.

Approach 1: The level of robustness can be increased by increasing the embedding strength for textured images while maintaining the level of decomposition $(L=2)$.

Approach 2: An alternative approach is to reduce the level of decomposition for textured images for watermarking while maintaining embedding strength $(k=2)$.

Both the approaches can maintain the desired level of robustness. Approach 2 is slightly better in terms of PSNR obtained. This can be seen as follows.

In approach 1, required higher embedding strength reduces the PSNR. While in approach 2, modifications in major frequency components at level-1 also reduce the PSNR. If the value of PSNR is maintained equal in both the approaches with adjustment of $k$, the value of $\mathrm{NC}$ will be slightly poorer in case of approach 1. At level-2 decomposition, the size of watermark embedding plane is smaller. This smaller size of coefficient matrix creates poor NC values as correlation is also affected by the size of data matrices. This slight reduction in NC of approach 1 , can be overcome by further increase in embedding strength. But this will further reduce the PSNR. Therefore, to achieve same level of robustness, PSNR of approach 1 is slightly lower than that of approach 2 .

Therefore, approach 2 is adopted for textured images. The degradation in PSNR for textured images is not a major issue. Due to large high frequency contents available in textured images, human eyes are not able to discern this degradation.

The discussion above concludes that non-textured images should be watermarked at level 2 while textured images should be watermarked at level 1 to achieve desired level of robustness for both types of images. This motivates a system which can identify the type of images (textured or non-textured) and watermark it accordingly. The level selection algorithm based on edge histogram is proposed in next section.

\section{LEVEL SELECTION ALGORITHM}

In this section, approach 2 of previous section is described to maintain the desired level of robustness. The proposed level selection algorithm identifies the image type as to whether it is textured or non-textured and then applies either level-1 or level-2 watermarking accordingly. The scheme is outlined in figure 2 .

To classify the images based on the contents, the Edge Histogram Descriptor (EHD) of Mpeg-7 [22] is suitably adapted. For classification, the whole image is divided into 16 non-overlapping blocks in $4 \times 4$ and each image block is then further divided into $2 \times 2$ pixel blocks as shown in figure 3 .

From these $2 \times 2$ sub blocks, the local edge orientation is captured for the following cases: vertical, horizontal, diagonal45, diagonal135 and isotropic (non-orientation specific) types of orientations. These are shown in table 2 along with their detector operators.

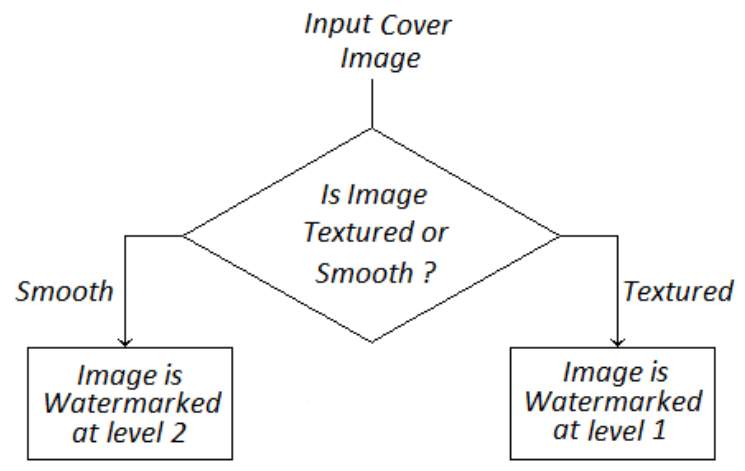

Fig 2. Adaptive Watermarking Algorithm based on image contents

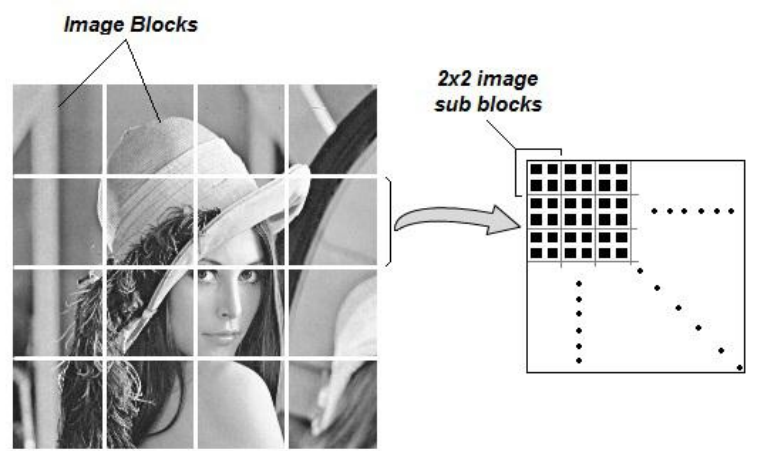

Fig 3. Image division for EHD calculation

The histogram for each sub-image represents the relative frequency of occurrence of the 5 types of edges in the corresponding sub-image. As a result, each local histogram contains 5 bins (Vertical, Horizontal, Diagonal45, Diagonal135 and Isotropic). Since there are 16 sub-images in the image, a total of $5 \times 16=$ 80 histogram bins are required to represent the image contents. 
Table 2. Five types of local edge orientations and their detectors

\begin{tabular}{|c|c|c|}
\hline Edge Type & Visual Representation & $\begin{array}{l}\text { Operator } \\
\text { Mask }\end{array}$ \\
\hline Vertical Edge & & {$\left[\begin{array}{ll}1 & -1 \\
1 & -1\end{array}\right]$} \\
\hline Horizontal Edge & & {$\left[\begin{array}{rr}1 & 1 \\
-1 & -1\end{array}\right]$} \\
\hline Diagonal $\left(45^{0}\right)$ & & {$\left[\begin{array}{cc}\sqrt{2} & 0 \\
0 & -\sqrt{2}\end{array}\right]$} \\
\hline Diagonal $\left(135^{0}\right)$ & & {$\left[\begin{array}{cc}0 & \sqrt{2} \\
-\sqrt{2} & 0\end{array}\right]$} \\
\hline $\begin{array}{l}\text { Non-Orientation Type } \\
\text { Edges }\end{array}$ & & {$\left[\begin{array}{rr}2 & -2 \\
-2 & 2\end{array}\right]$} \\
\hline
\end{tabular}

The edge orientation $(E O)$ of a $2 \times 2$ sub block is captured by applying all five detectors as follows.

$$
E O_{\text {type }}=\left|\sum_{k=0}^{3} a_{k} \cdot d_{k}\right|
$$

Where, $\left[a_{k}\right]=\left[\begin{array}{ll}a_{0} & a_{1} \\ a_{2} & a_{3}\end{array}\right]$ represents $2 \times 2$ image sub block and $\left[d_{k}\right]=\left[\begin{array}{ll}d_{0} & d_{1} \\ d_{2} & d_{3}\end{array}\right]$, represents the edge detector. For one image block, by applying all these five operators on a $2 \times 2$ image sub block, the five values $E O_{v}, E O_{h}$, $E O_{d 45}, E O_{d 135}$ and $E O_{i s o}$ are obtained and maximum of these five is compared with a threshold value $\left(T_{h}\right)$ to find the dominant edge orientation as, $\left(E O_{\text {dominant }}\right)=$ $\max \left(E O_{v}, E O_{h}, E O_{d 45}, E O_{d 135}, E O_{i s o}\right)>T_{h}$.

The count of corresponding bin is increased by one and it is repeated for all $2 \times 2$ sub blocks. Thus a first image block out of total 16 is represented by 5 bins as $(b 0, b 1, b 2, b 3, b 4)$. This process is repeated for all remaining 15 image blocks getting their 5 bins representation. For example, $2^{\text {nd }}$ block representation is $(b 5, b 6, b 7, b 8, b 9)$ and last block representation is $(b 75, b 76, b 77, b 78, b 79)$. From this five value representation of all 16 image blocks, a matrix is prepared as shown in (2).

AllBins $=\left[\begin{array}{ccccc}b 0 & b 1 & b 2 & b 3 & b 4 \\ b 5 & b 6 & b 7 & b 8 & b 9 \\ \cdot & \cdot & \cdot & \cdot & \cdot \\ \cdot & \cdot & \cdot & \cdot & \cdot \\ b 75 & b 76 & b 77 & b 78 & b 79\end{array}\right]$

The value of GlobalBinAvg is computed by taking column wise and row wise mean of matrix AllBins as,

$$
\text { GlobalBinAvg }=\operatorname{mean}(\operatorname{mean}(\text { AllBins }))
$$

In the proposed scheme, the value of GlobalBinAvg is taken as decision level for finding whether an image is textured or non-textured. To validate the results of image classification algorithm based on image contents, several textured and non-textured images are taken for experiment. For this purpose, total 107 images of size $512 \times 512$ of both the categories are collected from various sources including standard image databases and Word Wide Web. The results of image separation on the basis of their GlobalBinAvg are shown in table 3 and in figure 4 .

In figure 4, the mean values of GlobalBinAvg is also shown for both the categories of the images. Results shown in table 3 and figure 4 show the classifications of selected images on the basis of their edge histograms. There may be the possibility that some images lie on the verge of classification. This condition can lead to missclassification of the images. But this is not the problem because in this scheme, the objective is not to implement the precise classification of images. The simple edge histogram based algorithm is sufficient to facilitate the proper watermarking of smooth and textured images to achieve better robustness.

Table 3. Results of Image classification based on image contents

\begin{tabular}{lcccc}
\hline \multirow{2}{*}{ Image Type } & No. of & \multicolumn{3}{c}{ GlobalBinAvg } \\
\cline { 3 - 5 } & Images & Min. & Max. & Avg. \\
\hline $\begin{array}{l}\text { Smooth } \\
\text { (Low Frequency) }\end{array}$ & 107 & 0.00 & 87.09 & 14.19 \\
$\begin{array}{l}\text { Textured } \\
\text { (High Frequency) }\end{array}$ & 107 & 122.18 & 643.15 & 300.59 \\
\hline
\end{tabular}

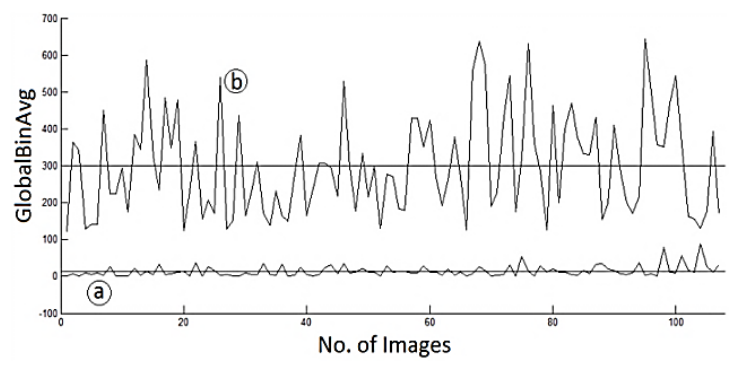

Fig 4. (a) GlobalBinAvg profile of smooth images, (b) GlobalBinAvgprofile of textured images

\section{WATERMARKING SCHEME}

The spread spectrum watermarking [6] is known to be robust against various types of attacks. In this paper, spread spectrum watermarking is used for watermark embedding and extraction for both types of images. Watermark embedding and extraction algorithms are explained in next subsections.

\section{A. Watermark Embedding Algorithm}

The steps of the proposed embedding algorithm are as follows.

Input: A grayscale image (I) of type uint8 and of size $M \times M$ and a binary watermark $(W)$. 
Output: Watermarked Image $\left(I_{w}\right)$.

1. The input image $(I)$ is categorized into either textured or smooth image by the level selection algorithm described. If image is textured then decomposition level $N=1$ otherwise $N=2$ for smooth images.

2. Perform N-level wavelet decomposition of input image $(I)$ to obtain four coefficients matrices $c A_{N}, c H_{N}, c V_{N}$ and $c D_{N}$ of size $\frac{M}{2^{N}} \times \frac{M}{2^{N}}$.

3. Select high frequency diagonal wavelet coefficient matrix $c D_{N}$ for watermark embedding.

4. Select a seed to generate a Pseudo Random Sequence $(P R S)$ of size equal to the size of frequency band $c D_{N}$ and modify it to obtain matrix $P N$ using the relation, $P N=R_{1} \times\left(P R S-R_{2}\right)$, where, $R_{1}=2$ and $R_{2}=$ 0.5 .

5. If watermark bit is 0 (Black) then modify the $c D_{N}$ wavelet coefficients as, $c D^{\prime}{ }_{N}=c D_{N}+k \cdot P N$, where, $k$ isembedding strength. If watermark bit is 1 (white) then wavelet coefficients are left unchanged.

6. Repeat the step 4 and 5 for all ' 0 ' watermark bits with a newly generated $P N$ sequence for each bit.

7. Take modified $c D^{\prime}{ }_{N}$ to its original position and take inverse DWT to get watermarked image $\left(I_{w}\right)$.

8. Compute the PSNR for $I$ and $I_{w}$ to determine the change in host image.

The embedding algorithm is shown in figure 5 .

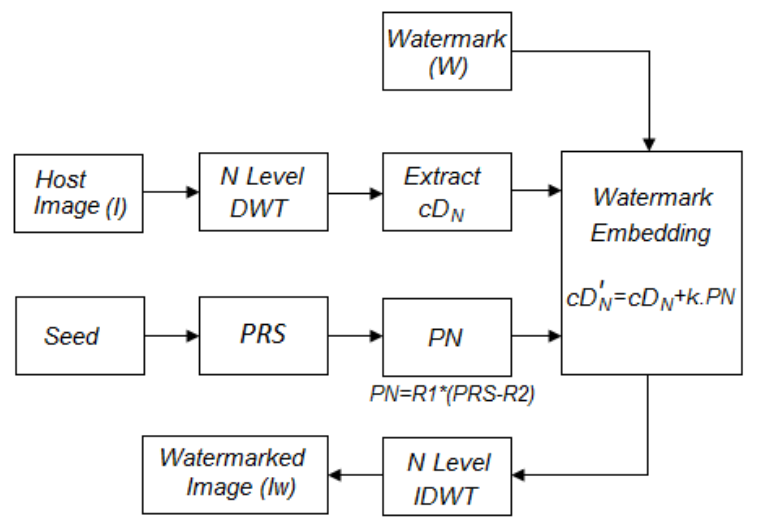

Fig 5. Watermark Embedding Algorithm for content based watermarking

\section{B. Watermark Extraction Algorithm}

Followings are the steps of watermark extraction, Input: Watermarked Image $\left(I_{w}\right)$ and Seed value. Output: Extracted Watermark $\left(W_{R}\right)$.

1. Decompose the watermarked image $\left(I_{w}\right)$ in $N$ levels and obtain the modified coefficients matrix $c D^{\prime}{ }_{N}$.

2. Generate the same random sequence $(P R S)$, which was generated in embedding process using same seed value and convert the random sequence into $P N=$ $R_{1} \times\left(P R S-R_{2}\right)$ with $R_{1}=2$ and $R_{2}=0.5$.

3. Compute the correlation coefficients between random sequence $(P N)$ and the modified coefficient matrix $c D^{\prime}{ }_{N}$ as $r=\operatorname{corr} 2\left(P N, c D^{\prime}{ }_{N}\right)$. Repeat step 2 and 3 for all watermark bits and compute all correlation values $r$.

4. Compute the mean correlation value $(T=$ mean $(r))$ and initialize a row matrix $\left(W^{\prime}\right)$ having all values ' 1 ' equivalent to the size of the watermark.

5. For every watermark bit, compare $r$ with $T$ and modify the $W^{\prime}$ as follows,

$$
W^{\prime}= \begin{cases}0, & r>T \\ 1, & \text { otherwise }\end{cases}
$$

6. Reshape the row matrix $W^{\prime}$ into a matrix of size of original watermark matrix $(W)$ to get recovered watermark $\left(W_{R}\right)$.

7. Compute the $\mathrm{NC}$ between original watermark (W) and recovered watermark $\left(W_{R}\right)$ to observe the quality of extracted watermark.

The watermark extraction algorithm is shown below graphically in figure 6.

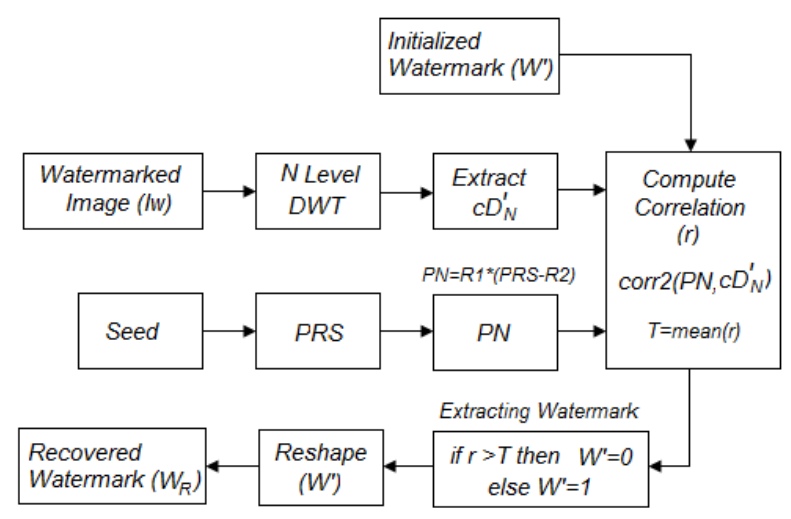

Fig 6. Watermark Extraction Algorithm for content based watermarking

\section{EXPERIMENTAL RESULTS AND ANALYSIS}

In this section, the proposed methodology is tested on various textured and non-textured images. These test images are already shown in figure 1 . All test images are grayscale and of size $512 \times 512$. The binary watermark used is shown in figure 7.

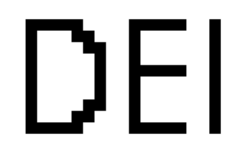

Fig 7. Binary Watermark $(21 \times 12)$

While computing GlobalBinAvg of equation 3, the threshold value $\left(T_{h}\right)$ is taken as 100 . For the purpose of automatic wavelet decomposition level selection, the value of GlobalBinAvg is chosen to be 120 as decision level.

This decision level will categorize the input host images as textured and non-textured images. The input images, those having GlobalBinAvg below 120 will be categorized as smooth images, while those having GlobalBinAvg above 120 will be considered as textured 
images. The decision level 120 is derived empirically by computing GlobalBinAvg with amount of texture variation in input host image as shown in table 4.

Table 4. Watermarking evaluation with texture variation

\begin{tabular}{|c|c|c|c|c|c|}
\hline \multirow[b]{2}{*}{ Images } & \multicolumn{2}{|c|}{ Level 1} & \multicolumn{2}{|c|}{ Level 2} & \multirow{2}{*}{$\begin{array}{c}\text { Global } \\
\text { Bin } \\
\text { Avg }\end{array}$} \\
\hline & PSNR & $\mathrm{NC}$ & PSNR & $\mathrm{NC}$ & \\
\hline \multicolumn{6}{|c|}{ Image: $512 \times 512$, Texture: $0 \times 0(0.00 \%)$} \\
\hline & 30.48 & 1.00 & 37.04 & 1.00 & 29.53 \\
\hline \multicolumn{6}{|c|}{ Texture: $64 \times 64(1.53 \%)$} \\
\hline & 31.71 & 1.00 & 38.20 & 1.00 & 34.05 \\
\hline \multicolumn{6}{|c|}{ Texture: $128 \times 128(6.25 \%)$} \\
\hline & 31.76 & 1.00 & 38.47 & 1.00 & 47.18 \\
\hline \multicolumn{6}{|c|}{ Texture: $192 \times 192(14.06 \%)$} \\
\hline & 31.79 & 1.00 & 38.47 & 1.00 & 69.00 \\
\hline \multicolumn{6}{|c|}{ Texture: $256 \times 256(25.00 \%)$} \\
\hline & 31.90 & 1.00 & 38.47 & 1.00 & 100.18 \\
\hline \multicolumn{6}{|c|}{ Texture: $320 \times 320(39.06 \%)$} \\
\hline & 31.90 & 1.00 & 38.54 & 0.9832 & 138.61 \\
\hline \multicolumn{6}{|c|}{ Texture: $384 \times 384(56.25 \%)$} \\
\hline & 31.90 & 1.00 & 38.54 & 0.9750 & 185.94 \\
\hline \multicolumn{6}{|c|}{ Texture: $448 \times 448(76.56 \%)$} \\
\hline & 31.90 & 1.00 & 38.54 & 0.9589 & 241.16 \\
\hline \multicolumn{6}{|c|}{ Texture: $512 \times 512(100.00 \%)$} \\
\hline & 32.09 & 1.00 & 38.54 & 0.8530 & 302.68 \\
\hline
\end{tabular}

All images in table 4 are watermarked with $k=2$ using both level-1 and level-2 decomposition with Bior6.8 wavelet. This wavelet is chosen because Bior6.8 is a symmetric, biorthogonal wavelet with linear phase and gives better reconstruction with minimum error. The
Peak Signal to Noise Ratio (PSNR) is calculated in $\mathrm{dB}$ between the watermarked image and original image. Normalized Correlation Coefficient (NC) between recovered watermark and original watermark is calculated under no attack condition.

From table 4, it can be observed that when the value of GlobalBinAvg is 138.61 (39.06\% texture), watermark recovery in level 2 is degraded with NC lesser than 1 . Therefore, it is better to choose level 1 watermarking to improve watermark extraction.

Similarly when the value of GlobalBinAvg is 100.18 , level-2 watermarking performs well in watermark extraction. Thus, an intermediate value of 120 is chosen as decision level for automatic level selection for textured and non-textured images aiming at better watermark extraction. The analysis shown in table 4 is done for $512 \times 512$ image size. For an image half of its size, the decision level can be down scaled by 0.5 and similarly for image double of its size, it can be up scaled by 2 assuming that the amount of texture is proportional to the image size. It can be seen from table 4 that although decomposition at level 1 improves watermark recovery for textured images, the value of PSNR reduces. This is not a big problem as already discussed because due to more texture available in the image, this small degradation in image is almost imperceptible to the human eyes.

The performance of watermarking scheme along with level selection algorithm is tested under various attacks such as JPEG compression, Cropping, Noise addition, Filtering etc. and the results are shown in the tables 5 to 11.

The shaded areas in tables 5 to 11 show the preferred level watermarking for smooth and textured images depending on the value of GlobalBinAvg. The value of $\mathrm{NC}$ calculated between original and recovered watermark clearly shows that in all attacks the robustness is better for smooth images if they are watermarked at level 2 while it is better for textured images, if they are watermarked at level 1 . The only exception is the case of blurring attack, where level 2 watermarking is more robust for textured images. This happens because, blurring attack removes high frequency details of the textured images, therefore, making them in the category of smooth images.

Table 5. Comparison of NC under JPEG Compression $(\mathrm{Q}=30)$

\begin{tabular}{lccccc}
\hline \multirow{2}{*}{ Image } & \multirow{2}{*}{$\begin{array}{c}\text { Global } \\
\text { BinAvg }\end{array}$} & \multicolumn{2}{c}{ Level1 } & \multicolumn{2}{c}{ Level2 } \\
\cline { 3 - 6 } & & PSNR & NC & PSNR & NC \\
\hline Lena & 8.20 & 30.71 & 0.6112 & 37.53 & 0.9669 \\
Pepper & 29.53 & 30.71 & 0.8157 & 36.98 & 0.9916 \\
Boat & 30.64 & 30.64 & 0.7562 & 37.33 & 0.9916 \\
Camera & 84.74 & 31.39 & 0.8293 & 38.08 & 0.9832 \\
Man & 363.26 & 31.20 & 0.8686 & 37.99 & 0.8498 \\
D15 & 260.71 & 31.37 & 0.9353 & 37.98 & 0.8868 \\
D20 & 364.61 & 31.70 & 0.8960 & 38.27 & 0.8906 \\
D84 & 402.08 & 31.64 & 0.8741 & 38.15 & 0.7529 \\
D110 & & & & & \\
\hline
\end{tabular}


Table 6. Comparison of NC under Salt \& Pepper Attack (Strength=0.1)

\begin{tabular}{lccccc}
\hline \multirow{2}{*}{ Image } & Global & \multicolumn{2}{c}{ Level1 } & \multicolumn{2}{c}{ Level2 } \\
\cline { 3 - 6 } & BinAvg & PSNR & NC & PSNR & NC \\
\hline Lena & 8.20 & 30.71 & 1.0000 & 37.53 & 0.8835 \\
Pepper & 29.53 & 30.71 & 1.0000 & 36.98 & 0.8338 \\
Boat & 30.64 & 30.64 & 1.0000 & 37.33 & 0.8363 \\
Camera Man & 84.74 & 31.39 & 1.0000 & 38.08 & 0.8060 \\
D15 & 363.26 & 31.20 & 0.9916 & 37.99 & 0.7433 \\
D20 & 260.71 & 31.37 & 0.9916 & 37.98 & 0.8102 \\
D84 & 364.61 & 31.70 & 1.0000 & 38.27 & 0.7992 \\
D110 & 402.08 & 31.64 & 1.0000 & 38.15 & 0.6712 \\
\hline
\end{tabular}

Table 7. Comparison of NC under Gaussian Noise Attack

$$
\left(\mu=0, \quad \sigma^{2}=0.003\right)
$$

\begin{tabular}{lccccc}
\hline \multirow{2}{*}{ Image } & Global & \multicolumn{2}{c}{ Level1 } & \multicolumn{2}{c}{ Level2 } \\
\cline { 3 - 6 } & BinAvg & PSNR & NC & PSNR & NC \\
\hline Lena & 8.20 & 30.71 & 1.0000 & 37.53 & 0.8800 \\
Pepper & 29.53 & 30.71 & 1.0000 & 36.98 & 0.9110 \\
Boat & 30.64 & 30.64 & 1.0000 & 37.33 & 0.9035 \\
Camera Man & 84.74 & 31.39 & 0.9916 & 38.08 & 0.9174 \\
D15 & 363.26 & 31.20 & 1.0000 & 37.99 & 0.7628 \\
D20 & 260.71 & 31.37 & 1.0000 & 37.98 & 0.8504 \\
D84 & 364.61 & 31.70 & 1.0000 & 38.27 & 0.7963 \\
D110 & 402.08 & 31.64 & 0.9916 & 38.15 & 0.6771 \\
\hline
\end{tabular}

Table 8. Comparison of NC under Sharpening Attack (Mask= [-1 -1 -1;-1 $9-1 ;-1-1-1]$ )

\begin{tabular}{lllccc}
\hline \multirow{2}{*}{ Image } & \multirow{2}{*}{$\begin{array}{c}\text { Global } \\
\text { BinAvg }\end{array}$} & \multicolumn{2}{c}{ Level1 } & \multicolumn{2}{c}{ Level2 } \\
\cline { 3 - 6 } & PSNR & NC & PSNR & NC \\
\hline Lena & 8.20 & 30.71 & 1.0000 & 37.53 & 1.0000 \\
Pepper & 29.53 & 30.71 & 1.0000 & 36.98 & 1.0000 \\
Boat & 30.64 & 30.64 & 1.0000 & 37.33 & 1.0000 \\
Camera Man & 84.74 & 31.39 & 1.0000 & 38.08 & 1.0000 \\
D15 & 363.26 & 31.20 & 1.0000 & 37.99 & 0.8624 \\
D20 & 260.71 & 31.37 & 1.0000 & 37.98 & 0.9916 \\
D84 & 364.61 & 31.70 & 1.0000 & 38.27 & 0.8979 \\
D110 & 402.08 & 31.64 & 1.0000 & 38.15 & 0.7726 \\
\hline
\end{tabular}

Table 9. Comparison of NC under Median Filtering Attack $(3 \times 3$ Mask $)$

\begin{tabular}{lllccc}
\hline \multirow{2}{*}{ Image } & Global & \multicolumn{2}{c}{ Level1 } & \multicolumn{2}{c}{ Level2 } \\
\cline { 3 - 6 } & BinAvg & PSNR & NC & PSNR & NC \\
\hline Lena & 8.20 & 30.71 & 0.9052 & 37.53 & 0.9833 \\
Pepper & 29.53 & 30.71 & 0.7760 & 36.98 & 0.9916 \\
Boat & 30.64 & 30.64 & 0.8007 & 37.33 & 0.9916 \\
Camera Man & 84.74 & 31.39 & 0.7760 & 38.08 & 1.0000 \\
D15 & 363.26 & 31.20 & 0.9510 & 37.99 & 0.7430 \\
D20 & 260.71 & 31.37 & 0.8979 & 37.98 & 0.7324 \\
D84 & 364.61 & 31.70 & 0.8172 & 38.27 & 0.8221 \\
D110 & 402.08 & 31.64 & 0.6411 & 38.15 & 0.6182 \\
\hline
\end{tabular}

Table 10. Comparison of NC under Blurring Attack $(3 \times 3$ Averaging Mask $)$

\begin{tabular}{llllll}
\hline \multirow{2}{*}{ Image } & Global & \multicolumn{2}{c}{ Level1 } & \multicolumn{2}{c}{ Level2 } \\
\cline { 3 - 6 } & BinAvg & PSNR & NC & PSNR & NC \\
\hline Lena & 8.20 & 30.71 & 0.6712 & 37.53 & 1.0000 \\
Pepper & 29.53 & 30.71 & 0.5994 & 36.98 & 1.0000 \\
Boat & 30.64 & 30.64 & 0.5398 & 37.33 & 1.0000 \\
Camera Man & 84.74 & 31.39 & 0.7004 & 38.08 & 1.0000 \\
D15 & 363.26 & 31.20 & 0.6548 & 37.99 & 0.8741 \\
D20 & 260.71 & 31.37 & 0.6258 & 37.98 & 0.9277 \\
D84 & 364.61 & 31.70 & 0.5600 & 38.27 & 0.9201 \\
D110 & 402.08 & 31.64 & 0.5046 & 38.15 & 0.6969 \\
\hline
\end{tabular}

Table 11. Comparison of NC under Cropping Attack (1/4th upper left is cut)

\begin{tabular}{lllllc}
\hline \multirow{2}{*}{\multicolumn{1}{c}{ Image }} & Global & \multicolumn{2}{c}{ Level1 } & \multicolumn{2}{c}{ Level2 } \\
\cline { 3 - 6 } & BinAvg & PSNR & NC & PSNR & NC \\
\hline Lena & 8.20 & 30.71 & 1.0000 & 37.53 & 1.0000 \\
Pepper & 29.53 & 30.71 & 1.0000 & 36.98 & 1.0000 \\
Boat & 30.64 & 30.64 & 1.0000 & 37.33 & 1.0000 \\
Camera Man & 84.74 & 31.39 & 1.0000 & 38.08 & 1.0000 \\
D15 & 363.26 & 31.20 & 1.0000 & 37.99 & 0.8960 \\
D20 & 260.71 & 31.37 & 1.0000 & 37.98 & 0.9832 \\
D84 & 364.61 & 31.70 & 1.0000 & 38.27 & 0.9264 \\
D110 & 402.08 & 31.64 & 1.0000 & 38.15 & 0.7298 \\
\hline
\end{tabular}

\section{CONCLUSIONS}

In this paper, an adaptive robust spread spectrum method of digital image watermarking in wavelet domain is proposed. The algorithm automatically selects the level of wavelet decomposition based on image contents to provide maximum robustness to both textured and nontextured images. The proposed scheme is tested for various types of images. The results show that there is good improvement in the quality (Correlation Coefficients) of recovered watermark under various attacks for textured images if they are watermarked at level 1 . Though the watermarking at level 1 reduces the PSNR value of textured images slightly but better robustness is achieved. The small degradation in the value of PSNR for textured images is not a problem as due to more intensity variations in textured image, this small degradation of image quality is almost insensitive to human eyes. Similarly for smooth images, level 2 decomposition works well, where better robustness with high PSNR is achieved. High PSNR is necessary in case of smooth images to avoid any noticeable visual artefacts in the image.

\section{REFERENCES}

[1] R. G. Van Schyndel, A. Z. Tirkel, C. F. Osborne, "A digital watermark", IEEE Proceedings ICIP, vol.2, pp. 8690, 1994.

[2] N. Nikolaidis, I. Pitas, "Copyright protection of images using robust digital signatures", IEEE International Conference on Acoustics, Speech Signal Processing, vol. 4, pp.2168-2171, May 1996.

[3] R. Wolfgang, E. Delp, "A watermark for digital image", IEEE Proceedings ICIP, Vol.3, pp.211-214, 1996.

[4] Emir Ganic, Scott D. Dexter, Ahmet M. Eskicioglu, "Embedding Multiple Watermarks in the DFT Domain using Low and High Frequency Bands", Proceedings on SPIE international conference on Security, Steganography, and Watermarking of Multimedia ContentsVII, Vol. 5681, 2005.

[5] Lin S. D., Chin Feng Chen, "A robust DCT-based watermarking for copyright protection", IEEE Transactions on Consumer Electronics, Vol. 46, Issue.03, pp. 415-421, August, 2000.

[6] I. J. Cox, Joe Kilian, F. Thomson Leighton, Talal Shamoon, "Secure Spread Spectrum Watermarking for Multimedia", IEEE Transactions on Image Processing, Vol. 6, No. 12, December 1997. 
[7] C. V. Serdean, M. Tomlinson, J. G. Wade, M. A. Ambroze, "Protecting Intellectual Rights: Digital WM in the Wavelet Domain", Proceedings of the IEEE International Workshop on Trends and Recent Achievements in Information Technology, 16-18 May 2002.

[8] C. Patvardhan, A. K. Verma, C. Vasantha Lakshmi, "A Comparative Analysis of Spread Spectrum Watermarking Technique in Wavelet Domain", Journal of Computer Science and Engineering (JCSE), U. K., Vol. 9, Issue 2, pp. 38-43, October 2011.

[9] C. Patvardhan, A. K. Verma, C. Vasantha Lakshmi, "A Robust Wavelet Packet Based Blind Digital Image Watermarking using HVS characteristics", International Journal of Computer Applications (IJCA), Vol. 36(9), pp. 06-12, December 2011.

[10] Nikita Kashyap, G. R. Sinha, "Image Watermarking Using 3-Level Discrete Wavelet Transform (DWT)", International Journal of Modern Education and Computer Science, MECS, Vol. 3, pp. 50-56, 2012.

[11] M. Natarajan, Y. Govindarajan, "Performance Comparison of single and multiple watermarking techniques", International Journal of Computer Network and Information Security, MECS, Vol. 7, pp. 28-34, 2014.

[12] M. S. Kankanhalli, Rajmohan, K. R. Ramakrishnan, "Content Based Watermarking of Images", Proceedings of the 6th ACM International Conference on Multimedia, pp. 61-70, Bristol, UK, September 1998.

[13] Xiaojun Qi, Ji Qi, "A robust content-based digital image watermarking scheme", Elsevier Journal of Signal Processing, Vol. 87, pp. 1264-1280, 2007.

[14] A. K. Parthasarathy, SubhashKak, "An Improved Method of Content Based Image Watermarking", IEEE Transactions on Broadcasting, Vol. 53, No. 02, pp. 468479, 2007.

[15] Sviatoslav Voloshynovskiy, Alexander Herrigel, Nazanin Baumgaertner, Thierry Pun, "A Stochastic Approach to Content Adaptive Digital Image Watermarking", Proceedings of 3rd Springer International Workshop on Information Hiding (IH), pp. 211-236, 1999.

[16] Mauro Barni, Franco Bartolini, Alessandro Piva, "Improved Wavelet-Based Watermarking Through PixelWise Masking", IEEE Transactions on Image Processing, Vol. 10, No. 05, pp. 783-791, 2001.

[17] Zhihua Xie, Shengqian Wang, Lixin Gan, Lin Zhang, Zhenghua Shu, "Content Based Image Watermarking in the Ridgelet Domain", IEEE International Symposium on Electronic Commerce and Security (ISECS), pp.877-881, 2008.

[18] F. Autrusseau, P. Le Callet, A. Ninassi, "A study of content based watermarking using an advanced HVS model", IEEE International Conference on Intelligent Information Hiding and Multimedia Signal Processing, pp. 485-488, 2007.

[19] Chih-Chin Lai, Chi-Feng Chan, Chen-Sen Ouyang, HuiFen Chiang, "A Robust Feature-Based Image Watermarking Scheme ", Proceedings of 4th IEEE International Conference on Software Engineering, Artificial Intelligence, Networking and Parallel/Distributed Computing (SNPD), pp. 581-585, July 2013.

[20] Chun-Hung Chena, Yuan-Liang Tangb, Chih-Peng Wangb, Wen-Shyong Hsieha, "A robust watermarking algorithm based on salient image features", Optik International Journal for Light and Electron Optics, Elsevier, Volume 125, Issue 3, pp. 1134-1140, Feb. 2014.
[21] Brodatz Texture Database, Available on http://www.ux.uis.no/ tranden/brodatz.html.

[22] Chee Sun Won, Dong Kwon Park, and Soo-Jun Park, "Efficient Use of MPEG-7 Edge Histogram Descriptor", ETRI Journal, Volume 24, Number 1, February 2002.

\section{Authors' Profiles}

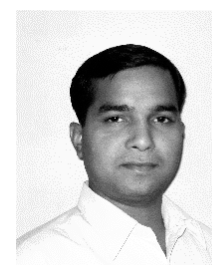

Dr. A. K. Verma is working in Hindustan Institute of Technology and Management, Agra, UP, India as Associate Professor in the department of Electrical and Electronics Engineering. He obtained his B.Sc.-Engg. in Electrical Engineering in year 2000 and M.Tech. in Engg. Systems in year 2002 from Dayalbagh Educational Institute (DEI). He was awarded with Gold Medals in both B.Sc.-Engg. and M.Tech. for securing highest marks. He has obtained his Ph.D. in March 2014 from DEI. His areas of research is wavelets and their applications in Signals and Image Processing. He has published more than 25 research papers in various international journals and conferences of repute. He is also reviewer of few international conferences. Dr. Verma is life member of ISTE, India.

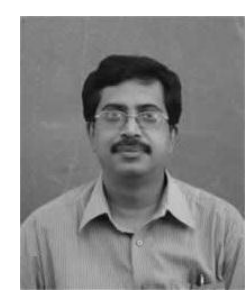

Prof. C. Patvardhan is working in the Dayalbagh Educational Institute (DEI), Agra, UP, India as Professor in Electrical Engineering department. He obtained his B.Sc. (Engg.) from DEI in 1987, M.Tech. from IISc, Bangalore in Computer Science in 1989 and his Ph.D. in 1994. He has published more than 250 papers in Journals and Proceedings of Conferences and has won 20 Best Paper Awards. He has also published one book and has been an Editor of two Conference proceedings. He has been the PI and Co-PI of several funded R\&D projects. His current research interests are Quantum and Soft Computing, Image Processing and he is a reviewer for International Journals and Conferences. He is a life member of Computer Society of India, Systems Society of India and Indian Science Congress Association and a Fellow of United Writers Association of India, Chennai.

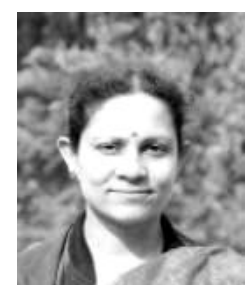

Dr. C. Vasantha Lakshmi is working in the Dayalbagh Educational Institute, Agra, UP, India as an Associate Professor in the Department of Physics and Computer Science. She obtained her B.Tech. (ECE) from JNTU in 1992, M.Tech. (CS) from Central University, Hyderabad in 1994 and Ph.D. from DEI, Dayalbagh in 2003. She has been the PI and Co-PI of several funded research projects. She has authored one book and has more than 40 publications in Journals and Conferences. Her research has been recognized by several awards including the Indian Science Congress Young Scientist Award in 2004 and Systems Society of India Young System Scientist Award in 2009. Her research focuses on Image Processing and Pattern Recognition. She is a member of the IEEE and the IEEE Computer Society.

Manuscript received Month Date, Year; revised Month Date, Year; accepted Month Date, Year. 